\title{
42 THE ROLE OF TISSUE STIFFNESS IN PREDICTING THE IMMUNOTHERAPY RESPONSE IN HEPATOCELLULAR CARCINOMA
}

Betul Gok Yavuz*, Elshad Hasanov, Lianchun Xiao, Yehia Mohamed, Sunyoung Lee, Asif Rashid, Ahmed Kaseb, Aliya Qayyum. UT MD Anderson Cancer Center, Houston, TX, USA

Background Currently, there is no standard biomarker that predict immunotherapy response in hepatocellular carcinoma (HCC). Here, we aim to investigate the role of tissue stiffness measured by magnetic resonance elastography (MRE) in predicting neoadjuvant immunotherapy response in patients with resectable HCC.

Methods This was a study of 15 patients with HCC treated with immune checkpoint blockade (ICB) therapy, nivolumab \pm ipilimumab, followed by surgical resection. HCC MRE assessment was performed at baseline and after 6 weeks of therapy. HCC stiffness $(\mathrm{kPa})$ was measured on MRE elastograms (liver stiffness maps). Baseline stiffness and changes in stiffness were compared with treatment response to ICB. Treatment response was defined as a tumor with more than $60 \%$ necrosis which was the major pathological response. Analysis was performed using descriptive statistics, Fisher's exact test, and Wilcoxon rank sum test; p-value $<0.05$ was considered statistically significant.

Results Fifteen patients were evaluable for MRE assessment. The median age was 67 years. Etiology of liver disease was NASH $(n=4), \operatorname{HCV}(n=3), \operatorname{HBV}(n=2)$ and unknown $(n=6)$. Three out of 15 patients (20\%) achieved a major pathological response (MPR). Median baseline HCC stiffness and change in stiffness were $4.6 \mathrm{kPa}$ and $-0.2 \mathrm{kPa}$, respectively. Among the 4 patients with stiffness increase, 3 (75\%) of them achieved MPR and 1 (25\%) did not achieve MPR. Among the patients without stiffness increase, none of them achieved MPR. Fisher's exact test indicates that increase in stiffness was associated with a higher chance to achieve MPR than patients without stiffness increase $(p=0.0088)$. Median baseline HCC stiffness for responders and non-responders was $6.8(5.4,9)$ $\mathrm{kPa}$ and $3.9(2.2,9.7) \mathrm{kPa}$, respectively $(\mathrm{p}=0.09)$. The median change in HCC stiffness for responders and non-responders was $1(1,1.4) \mathrm{kPa}$ and $-0.4(-2.2,0.7) \mathrm{kPa}$, respectively $(\mathrm{p}=0.02)$.

Conclusions Patients who achieved MPR inclined to have a higher baseline stiffness than patients who did not achieve MPR. Regarding the changes in stiffness between the two arms, patients with MPR group had a greater increase than that in the non-MPR group. In conclusion, baseline and change in MRE stiffness may be a useful biomarkers in predicting response to ICB therapy in HCC.

Ethics Approval This was an Institutional Review Board approved study (MDACC 2017-0972). All patients provided written informed consent.

http://dx.doi.org/10.1136/jitc-2021-SITC2021.042 